J. Appl. Cryst. (1974). 7, 188

\title{
Neutron Diffraction Patterns of Labelled Polymer Chains in the Bulk
}

\author{
By J. P. Cotton, B. Farnoux, J. S. Higgins, * G. Jannink and R. Ober $\dagger$ \\ DPh-G/PSRM CEN-Saclay, BP $n^{\circ} 2$ - 91190, Gif-sur-Yvette, France
}

The neutron diffraction patterns for labelled polymer chains in the bulk are investigated in the smallangle and the wide-angle scattering ranges. Using the principle of incompressibility, it is possible to take into account the environment of a labelled chain and to simulate the density-density response function of that chain by a computer program. In particular the contributions of the interference between the labelled and the unlabelled chains is calculated. This is carried out respectively for the isotropic and anisotropic situations. The calculation shows that a chain in the bulk has a 'Gaussian' configuration.

* Present address: I.L.L., 38042, Grenoble, France.

$\uparrow$ Laboratoire de la matière condensée, Collège de France.

J. Appl. Cryst. (1974). 7, 188

The Determination of the Conformation of Polymers in Bulk by Neutron Diffraction

\author{
By R. G. Kirste AND W. A. Kruse \\ Sonderforschungsbereich 41, D-65 Mainz, Chemische Institute, Germany (BRD) \\ AND J. SCHELTEN \\ Kernforschungsanlage Jülich, D-517 Jülich, Germany (BRD)
}

\begin{abstract}
Light and X-ray small-angle diffraction yield poor information on the shape of chain molecules in the bulk state. For this problem a new kind of proceeding has been established (R. G. Kirste, Jahresbericht 1969 des Sonderforschungsbereichs 41, Mainz 1970, p. 47). Dilute solid solutions of the ordinary polymer in the perdeuterated polymer are prepared. The neutron small-angle diffraction on these samples can be evaluated in a similar way to light and X-ray scattering on dilute solutions of polymers in low molecular compounds. One has to subtract the scattering of a polymer sample which contains the same amount of $\mathrm{D}$ and $\mathrm{H}$ but randomly distributed over all molecules. As a first example vitreous polymethylmethacrylate (PMMA) has been investigated. The molecular weights were $M=0.08,0.25,0.5$ and $1.0 \times 10^{6}$. With $M=0.25 \times 10^{6}$ at the concentrations $c=0 \cdot 3,0.6,0.9$ and $1.2 \%$ H-PMMA in D-PMMA Z Zimm plot has been obtained. The weight-average molecular weight from this plot is $M_{w}=230000$. The excess value of the chemical potential is zero. The $z$ average of the radius of gyration is $r_{z}=136 \AA$ which corresponds because of the non-uniqueness of $U=0.3$ to $r_{w}=121 \AA$. The uncertainty in $r_{w}$ and $r_{z}$ is about $\pm 10 \%$. The radius of gyration is proportional to the square root of $M$, but about 10 or $20 \%$ larger than the corresponding value in a low molecular $\theta$-solvent. Thus the unperturbed or nearly unperturbed random coil is the conformation of chain molecules in the amorphous solid state. Other current hypotheses such as the 'meander' structure or the collapsed-ball concept can now be excluded.
\end{abstract}

J. Appl. Cryst. (1974). 7, 188

Dimensions of Molecular Chains in the Bulk and Solutions

By H. Benoit, * J. P. Cotton, $\ddagger$ D. Decker, ${ }^{*}$ B. Farnoux,$\ddagger$ J. S. Higgins, ${ }^{*}$ G. Jannink, $\ddagger$ R. Ober $\dagger$ AND C. PICOT*

${ }^{*}$ C.R.M., 6 rue Boussingault, 67 Strasbourg, France

$\uparrow$ Laboratoire de la Matière Condensée, Collège de France, 11 Place M. Berthelot, 75005 Paris, France

$\$ D P h$-G/PSRM, CEN-Saclay, BP No. 2, 91190 Gif-sur-Yvette, France

The results of a comparative study of the dimensions of a flexible polymer (atactic polystyrene) in three environments are presented. Small-angle neutron scattering measurements were made on four deuterated polystyrene samples of different molecular masses in respectively a good solvent (carbon disulphide), a $\Theta$-solvent (Cyclohexane $36 \mathrm{C}$ ) and in the hydrogenous bulk polymer. The values of the radii of gyration and of the second virial coefficients are discussed. 